\title{
Encrusted Uropathy Associated with Cutanous Lesion and Renal Failure
} H. El Mortaji ${ }^{1 *}$, A. Chehboune ${ }^{1}$, B. Boutakioute ${ }^{1}$, M. Ouali Idrissi ${ }^{1}$, N. Cherif Idrissi Ganouni ${ }^{1}$, N. Elaatoul ${ }^{2}$, O. Hocar ${ }^{2}$, S. Amal ${ }^{2}$

${ }^{1}$ Radiology Department, Arrazi Hospital, CHU MED VI, Cadi Ayad University, Marrakech, Morocco

${ }^{2}$ Dermatology Department, Arrazi hospital, CHU MED VI, Cadi Ayad University, Marrakech, Morocco

Encrusted uropathy is a rare condition in which infection by ureas germs, most commonly Corynebacterium urealyticum, can lead to urinary tract calcification. Moreover, it usually occurs in immunocompromised patients, especially in renal transplants patients or patients with a history of previous urological procedures. The diagnosis is made easy thanks to the contribution of imagery, combined with the measurement of urinary $\mathrm{pH}$ and the isolation of the responsible germ. We describe the case of a 62 year old male patient with an alkaline encrusted uropathy in native kidneys associated with cutanous lesions and complicated with a renal failure and vein thrombosis.

Keywords: Encrusted uropathy, ultra sonography, CT, Corynebacterium.

Copyright $\odot$ (C) 2021 The Author(s): This is an open-access article distributed under the terms of the Creative Commons Attribution 4.0 International License (CC BY-NC 4.0) which permits unrestricted use, distribution, and reproduction in any medium for non-commercial use provided the original author and source are credited.

\section{INTRODUCTION}

Alkaline-encrusted uropathy is a severe chronic infection of urinary tract. It was first reported in 1914 [1]. More recently, alkaline-encrusted uropathy has been reported particularly in renal transplants or after surgical procedures of the urinary tract [2].

Corynebacterium urealyticumis is a common skin colonizer, seen in up to $30 \%$ of hospitalized elderly individuals who are receiving broad-spectrum antibiotics. This opathogen mainly causes acute cystitis, pyelonephritis, encrusted cystitis, and encrusted pyelitis $[2,3]$.

In our case, the patient presented with extensive cutanous lesions wich may explain the occurrence of this disease in native kidneys with no predisposing factors and make the particularity of this report.

\section{CASE RePORT}

We report the case of à 62 year old male patient who was first admitted for etiological assessment of à skin ulceration (figure 1). During his stay at the hospital, the patient presented with dysuria and a total hematuria. The biological investigations showed a renal failure and signs of infection with positive Corynebacterium blood cultures. The ultrasounds showed bilateral nephromegaly with a thickened and irregular blader wall (figure 2). The CT scan showed the mucosal encrustations of the bladder wall suggestive of encrusted cystitis (figure 3 ). There were associated a bilateral pyelitis and thrombosis of the renal veins (figure 4). The ertapenem was given as intravenous infusion in association with a good oral rehydration therapy. An improvement of his symptoms was noted with a restitution of his renal failure.

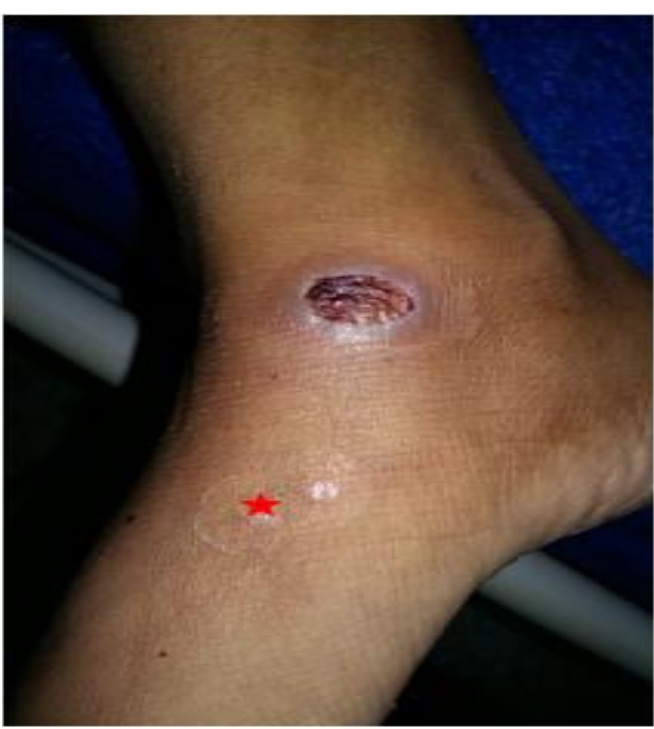

Fig-1: Skin ulceration located on the right foot with crusty borders with the evidence of a second totally crusted lesion (star) 


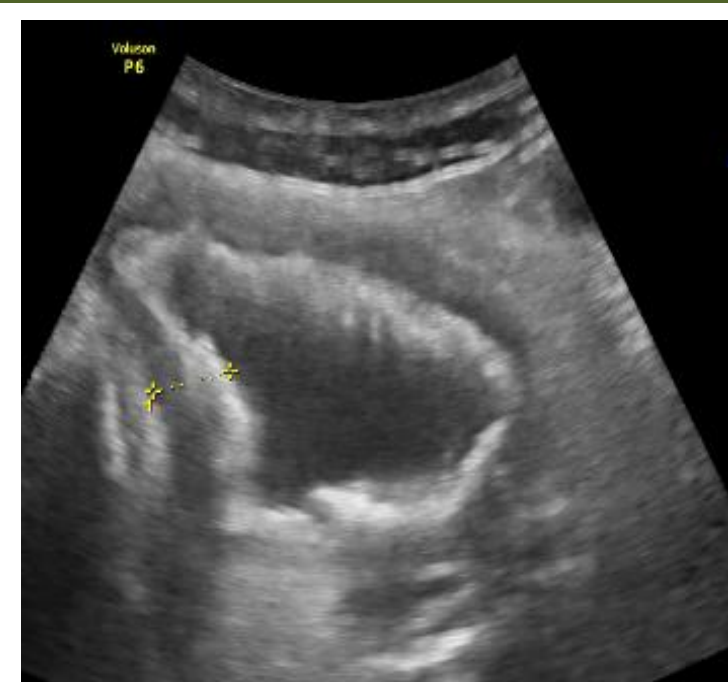

Fig-2: Irregular thickening with calcifications of the bladder wall

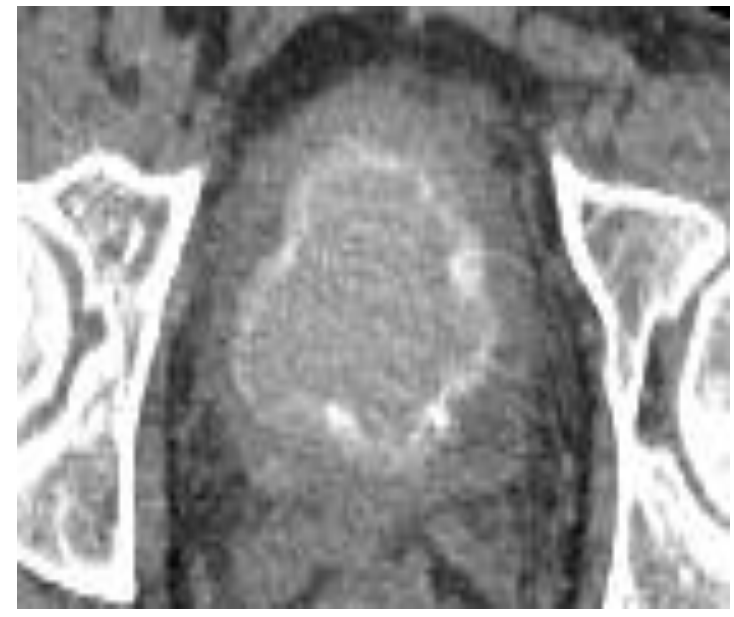

Fig-3: Irregular and heterogenous thickening of the bladder wall. The calcifications are located within the intern side of the bladder wall

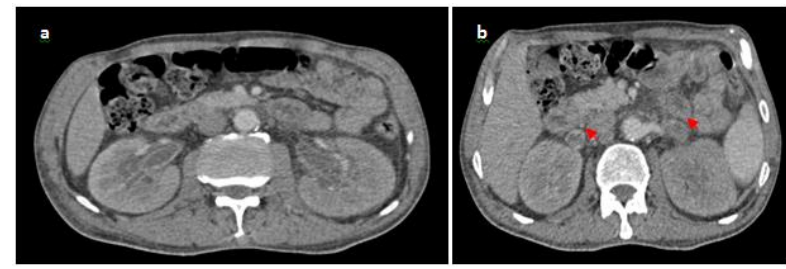

Fig-4: A: Bilateral thickening of the pyelic walls enhanced with the contrast associated with renal vein thrombosis (b)

\section{DISCUSSION}

Encrusted uropathy is usually caused by Corynebacterium urealyticum, a Gram-positive, slowgrowing, lipophilic, asaccharolytic, and usually multi drug-resistant organism with strong urease activity, which can infect the upper and lower urinary tract [4].

This bacterium is a urease-producing microorganism transforming urea into ammonia wich explains the modification of $\mathrm{pH}$ in urine that becomes alkaline. This leads to formation of struvite and calcium phosphate wich are responsible for wall encrustation and stone formation.

Previously reported cases of encrusted uropathies have occurred in patients with predisposing factors. Urological procedures, prolonged catheterization, or long-term hospitalization with broadspectrum antibiotic therapy have most commonly been reported as causative factors [5]. Encrusted uropathy has been observed in immunosuppressed patients, including renal transplant patients, and in those being treated for cancer or a systemic autoimmune disorders such as rheumatoid arthritis, systemic lupus erythematosus, or vasculitis [6, 7]. Rarely reported predisposing actors include neurologic bladder dysfunction and ectopic kidney [8].

In our case, the patient has a competent immune system and no history of urological procedures. Yet, he had extensive cutanous lesions and since the Corynebacterium urealyticum is a common skin colonizer; those lesions may be considered as a gateway to his urinary tract infection.

In urological literature on Corynebacterium urealyticum infections, there are many isolated clinical cases or small series $[9,10]$, but until today there are no conclusive data on its incidence. However because of an increasing number of renal transplants and other complex urologic procedures in an older or debilitated patient population, Sánchez-Martín and al [11] demonstrate the growing increase in cases of Corynebacterium urealyticum infections.

Encrusted cystitis usually presents with dysuria and suprapubic pain. Encrusted pyelitis can be minimally symptomatic for a long period. Fever is inconstant. Macroscopic hematuria with elimination of stones and an ammonia odor of the urine are strongly indicative of this infection. It may be also revealed by a clinical consequence such as acute obstruction or renal failure in patients with transplants [12].

The specific diagnosis of C. urealyticum infection may be missed by a standard urine culture and requires a prolonged incubation for more than 48 hours on special media. In some reported cases, this bacterium was not found because it was not specifically sought [13].

Imaging features is a non invasive way that could be helpful for the diagnosis. Alkaline-encrusted cystitis or pyelitis is defined by stone encrustation of the urinary tract wall, which can be depicted on an abdominal radiograph. In the bladder, when calcification outlines the bladder wall, the diagnosis is simple. In the upper urinary tract, when calcification is bulky or associated with free stones and could be mistaken for a staghorn calculus, the diagnosis can be 
more difficult [13]. Moreover, calcification of encrusted pyelitis may be radiolucent [12].

Sonography is also a useful diagnostic tool when calcifications lies in the bladder. However, in the caliceal system, this encrustation is difficult to differentiate from staghorn calculus. Therefore, CT appears to be the optimal technique to diagnose encrustation, particularly in the upper urinary tract [12].

The differential diagnosis includes schistosomiasis, tuberculosis, urothelial carcinoma, malacoplasia, cystophatie due to cyclophosphamide or mitomycin [14]. Treatment includes appropriate antimicrobial therapy, urine acidification, and surgical removal of encrustations. Corynebacterium is typically sensitive to glycopeptides, including vancomycin and teicoplanin. Acidification of the urine has a synergistic effect with antibiotics by dissolving the calcified encrustations and preventing the formation of additional encrustations. Urine is acidified with oral or topical acidic preparations. Application of a topical acidic solution through a catheter or nephrostomy tube is usually needed, especially at the start of treatment. Finally, surgical resection of encrustations via cystoscopy is usually necessary and often requires multiple procedures. Left untreated, encrusted cystitis and pyelitis can eventually lead to renal failure [15].

\section{Conlusion}

The clinical suspicion of encrusted uropathy should be more likely considered in renal transplant and immunocompromised patients. CT is the imaging test of choice. As the other urinary tract infections, encrusted uropathy can also be complicated with renal failure and venous thrombosis. Treatment is multimodal and personalized and should be start as fast as possible to avoid complications. Finaly the occurrence of urinary symptoms in old patients with extensive cutanous lesions should be considered carefully!

\section{REFERENCES}

1. François J. La cystite incrustée. J Urol Med Chir. 1914; 5: 35-52.

2. Chung SY, Davies BJ, O'Donnell WF. Mortality from grossly encrusted bilateral pyelitis, ureteritis, and cystitis by Corynebacterium group D2. Urology. 2003 Feb 1;61(2):463.

3. Anagnostou N, Siddins M, Gordon DL. Encrusted cystitis and pyelitis. Internal medicine journal. 2012 May 23;5(42):596-7.

4. Meria P, Desgrippes A, Arfi C, LE DUC AL. Encrusted cystitis and pyelitis. The Journal of urology. 1998 Jul 1;160(1):3-9.

5. Del Prete D, Polverino B, Ceol M, Vianello D, Mezzabotta F, Tiralongo E, Iafrate M, De Canale
E, Mengoli C, Valente M, Anglani F. Encrusted cystitis by Corynebacterium urealyticum: a case report with novel insights into bladder lesions. Nephrology Dialysis Transplantation. 2008 Aug 1;23(8):2685-7.

6. Soriano F, Tauch A. Microbiological and clinical features of Corynebacterium urealyticum: urinary tract stones and genomics as the Rosetta Stone. Clinical microbiology and infection. 2008 Jul 1;14(7):632-43.

7. Pagnoux C, Bérezné A, Damade R, Paillot J, Aouizerate J, Le Guern V, Salmon D, Guillevin L. Encrusting cystitis due to Corynebacterium urealyticum in a patient with ANCA-associated vasculitis: case report and review of the literature. InSeminars in arthritis and rheumatism 2011 Oct 1 (Vol. 41, No. 2, pp. 297-300). WB Saunders.

8. Guimarães LC, Soares SC, Albersmeier A, Blom J, Jaenicke S, Azevedo V, Soriano F, Tauch A, Trost E. Complete genome sequence of Corynebacterium urealyticum strain DSM 7111, isolated from a 9. year-old patient with alkaline-encrusted cystitis. Genome announcements. 2013 Jun 27;1(3).

9. Lieten S, Schelfaut D, Wissing KM, Geers C, Tielemans C. Alkaline-encrusted pyelitis and cystitis: an easily missed and life-threatening urinary infection. Case Reports. 2011 Jan 1;2011:bcr1220103613.

10. Curry CR, Saluja K, Das S, Thakral B, Dangle P, Keeler TC, Watkin WG. Encrusted cystitis secondary to Corynebacterium glucuronolyticum in a 57-year-old man without predisposing factors. Laboratory medicine. 2015 May 1;46(2):136-9.

11. Sánchez-Martín FM, López-Martínez JM, Kanashiro-Azabache A, Moncada E, Angerri-Feu $\mathrm{O}$, Millán-Rodríguez F, Villavicencio-Mavrich $\mathrm{H}$. Corinebacterium urealyticum: increased incidence of infection and encrusted uropathy. Actas Urológicas Españolas (English Edition). 2016 Mar $1 ; 40(2): 102-7$.

12. Thoumas D, Darmallaicq C, Pfister C, SavoyeCollet C, Sibert L, Grise P, Lemaitre L, Benozio M. Imaging characteristics of alkaline-encrusted cystitis and pyelitis. American Journal of Roentgenology. 2002 Feb;178(2):389-92.

13. Hertig A, Duvic C, Chretien Y, Jungers $P$, Grünfeld JP, Rieu P. Encrusted pyelitis of native kidneys. Journal of the American Society of Nephrology. 2000 Jun 1;11(6):1138-40.

14. Pollack HM, Banner MP, Martinez LO, Hodson CJ. Diagnostic considerations in urinary bladder wall calcification. American Journal of Roentgenology. 1981 Apr 1;136(4):791-7.

15. Tanaka T, Yamashita S, Mitsuzuka K, Yamada S, Kaiho Y, Nakagawa H, Arai Y. Encrusted cystitis causing postrenal failure. Journal of Infection and Chemotherapy. 2013 Dec 1;19(6):1193-5. 